\title{
Evolution of virial clouds-I: from surface of last scattering up to the formation of population-III stars
}

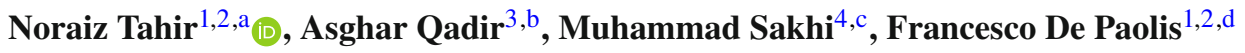 \\ ${ }^{1}$ Department of Mathematics and Physics "E. De Giorgi”, University of Salento, Via per Arnesano, 73100 Lecce, Italy \\ ${ }^{2}$ INFN, Sezione di Lecce, Via per Arnesano, 73100 Lecce, Italy \\ ${ }_{3}$ Abdus Salam School of Mathematical Sciences, Government College University Lahore, Lahore, Pakistan \\ ${ }^{4}$ Department of Physics, Quaid-e-Azam University, Islamabad, Pakistan
}

Received: 11 May 2021 / Accepted: 6 September 2021 / Published online: 17 September 2021

(C) The Author(s) 2021

\begin{abstract}
The analysis of WMAP and Planck CMB data has shown the presence of temperature asymmetries towards the halos of several galaxies, which is probably due to the rotation of clouds present in these halos about the rotational axis of the galaxies. It had been proposed that these are hydrogen clouds that should be in equilibrium with the CMB. However, standard theory did not allow equilibrium of such clouds at the very low CMB temperature, but it was recently shown that the equilibrium could be stable. This still does not prove that the cloud concentration and that the observed temperature asymmetry is due to clouds in equilibrium with the CMB. To investigate the matter further, it would be necessary to trace the evolution of such clouds, which we call "virial clouds", from their formation epoch to the present, so as to confront the model with the observational data. The task is to be done in two steps: (1) from the cloud formation before the formation of first generation of stars; (2) from that time to the present. In this paper we deal with the first step leaving the second one to a subsequent analysis.
\end{abstract}

\section{Introduction}

It had been proposed that a fraction $f$ in the form of molecular hydrogen clouds at exactly the CMB temperature in galactic halos may be a significant component of their dark matter [1]. To look for these chameleon clouds one suggestion was to look for an asymmetric Doppler shift in the CMB, due to the rotation of these clouds in the M31 halo [2]. The shift was observed first by analyzing WMAP [3] and then, more

\footnotetext{
a e-mail: noraiz.tahir@le.infn.it (corresponding author)

b e-mail: asgharqadir46@gmail.com

c e-mail: muhammad.saki@yahoo.com

de-mail: francesco.depaolis@le.infn.it
}

precisely, the Planck data [4]. This effect opened up a window to study galactic halos in more detail, and was thought to be a significant method to constrain the missing baryons [5]. In a series of papers the CMB data was used to trace the rotation of the halos of some nearby galaxies [6-10]. The data showed a temperature antisymmetry not only of the galactic disks but also of the galactic halos up to distances of hundreds of kpc from the galactic center, and the asymmetry was also used to study the rotational dynamics of the halos of some nearby spiral galaxies $[11,12]$. The fact that the temperature asymmetry was almost frequency independent strongly supports the Doppler shift explanation to observe the chameleon clouds in the halos. However, this did not prove that the clouds are made by pure molecular hydrogen, as originally proposed, since helium, dust and other heavier molecules may also be present, and they might contaminate these clouds with a significant fraction. These clouds were to survive on account of the virial temperature being in equilibrium with the CMB, we called them "virial clouds" [13]. It is suggested that no such equilibrium could exist at the current, very low, CMB temperatures, as there would be no mode that could be excited by the photons and the available clouds might collapse to form stars or other planetary objects [14]. Recently it was demonstrated that this equilibrium does arise on account of the translational mode, despite the extremely small probability, because of the size of the virial clouds and the time scales available for thermal equilibrium to be reached, so that the time required for thermalization is much less than that required for collapse [15].

Proving that virial clouds can exist does not prove that they do exist and play a significant role in the observed temperature asymmetry in the $\mathrm{CMB}$ towards some galaxies. To take the matter further we need to trace the evolution of these virial clouds from the time of their formation to the present, 
allowing for all the major qualitative changes that occurred during that time.

Virial clouds would have been formed at the time of the surface of last scattering (SLS) at $z=1100$ when the CMB temperature was about $3000 \mathrm{~K}$. At this stage recombination occurred and the CMB filled the Universe with a red, uniformly bright glow of blackbody radiation. With the passage of time the temperature decreased and the CMB shifted to the infrared and finally to the microwave region of the electromagnetic spectrum. To human eyes, the Universe would then have appeared as a completely dark place. A long period of time had to pass until the first objects collapsed, forming the population-III that shone in the universe with the first light ever emitted that was not part of the CMB [16]. In order to maintain the stability virial clouds must be in quasi-static equilibrium with the CMB since SLS. Obviously, when the clouds formed their chemical composition was the primordial one with about $75 \%$ atomic hydrogen and the rest helium. Afterwards, we expect a fast change of their composition as a consequence of the formation of population III stars. The question that arises is "When did the population-III form?" Because the primordial density fluctuations in the Universe are random, the question of when the very first star formed does not have a simple answer. The time when the first halo collapsed depends on how rare a fluctuation we are willing to consider. For example a $5 \sigma$ fluctuation in the density field can lead to the collapse of the halo and the formation of population-III at $z=30$ [17]. Using this large volume the population-III in the sky should be one formed from $8 \sigma$ fluctuations at $z=48$ when the temperature of the Universe was about $132 \mathrm{~K}$ [18]. Because of the qualitative changes that took place during and after the formation of population-III, we intend to model the evolution of these virial clouds in two steps: (1) from SLS at $z=1100$ when the temperature of the Universe was $3000 \mathrm{~K}$, up to the formation of population-III at $z=50$ when the temperature of the Universe was 137 $\mathrm{K}$; (2) from $z=50$ when the population-III were forming, and consequently the composition of the interstellar medium (ISM) changed [19] (and hence the clouds), to the present. It must be admitted that there would be significant changes during the second step, so it will be studied more clearly and in more detail later on.

In this paper we study the first step of the evolution of virial clouds, which begin at SLS about 380,000 years after the Big Bang at $z=1100$ when the Universe was very hot $\sim 3000$ $\mathrm{K}$, up to the formation of population-III at $z=50$ when the Universe was $137 \mathrm{~K}$. Since at the SLS the Universe consists of primordial abundance of atomic hydrogen and helium i.e. $\sim 75 \% \mathrm{H}$, and $\sim 25 \% \mathrm{He}$ by mass, this ratio should have remained almost the same till the formation of population III stars [16]. There were other molecules and atoms that would have been present at that time, which include deuterium, helium-3, lithium and molecular hydrogen [20,21], which could have contributed to the virial clouds. Molecular hydrogen played a role as a coolant in the clouds [24,25], and it would have formed in traces inside the clouds in the early stages. During the time span under study the ratio of molecular hydrogen to atomic hydrogen $n_{H_{2}} / n_{H}$ is $\approx 10^{-6}$ [22]. Moreover, the ratio of other elements like deuterium, helium-3, and lithium to atomic hydrogen is negligible [23]. The ratio of atomic hydrogen and helium would remain the same and these would be the main component to form the virial clouds during the period.

The plan of the paper is as follows: In Sect. 2 we will use the canonical distribution for obtaining the changing density, size and mass of a single fluid virial cloud from $\mathrm{z}=1100$ to $\mathrm{z}$ $=50$ (see Sect. 2.1). In the next section we extend our analysis to the two fluids model with $\mathrm{He}$ and $\mathrm{H}$ in the primordial mix (Sect. 2.2). Finally, in Sect. 3 we conclude with a discussion of the results.

\section{Canonical distribution function}

Since the clouds must be considered to be in thermal equilibrium because they are embedded in the heat bath of the CMB [26], we need to use the canonical distribution function for a fixed temperature and use the cooling of the heat bath to provide a quasi-equilibrium. This is justified because the changes are very slow compared to the scale of thermalization. One may wonder if quantum effects may not be significant and could affect the physical parameters of virial clouds. Since the present paper deals with temperatures above $137 \mathrm{~K}$, quantum effects are negligible, accounting for the cloud density. We will start by considering single fluid clouds and then go on to the two fluids case. Since the gas to be considered is either $\mathrm{H}$ or $\mathrm{He}$, it will be monatomic. For the monatomic gas the Hamiltonian incorporates only the translational mode. The clouds should start to form in the potential well of cold dark matter (CDM). As the clouds are thermalized the potential well will not cause them to collapse to form population-III stars [16], but will modify the physical parameters i.e. the mass, radius and central density.

\subsection{Single fluid}

For the sake of simplicity, we start our analysis by considering pure atomic hydrogen clouds. Since atomic hydrogen is monatomic, we can easily get the results by using the density distribution and Lane-Emden equation (obtained in [15]) with the boundary condition that the density is exactly zero at the edge of the cloud. The equations are

$$
\rho(r)=8 m^{5 / 2}\left(\frac{G \rho_{c}}{3 k_{B} T}\right)^{3 / 2} \exp \left(-\frac{G M(r) m}{r k_{B} T}\right),
$$




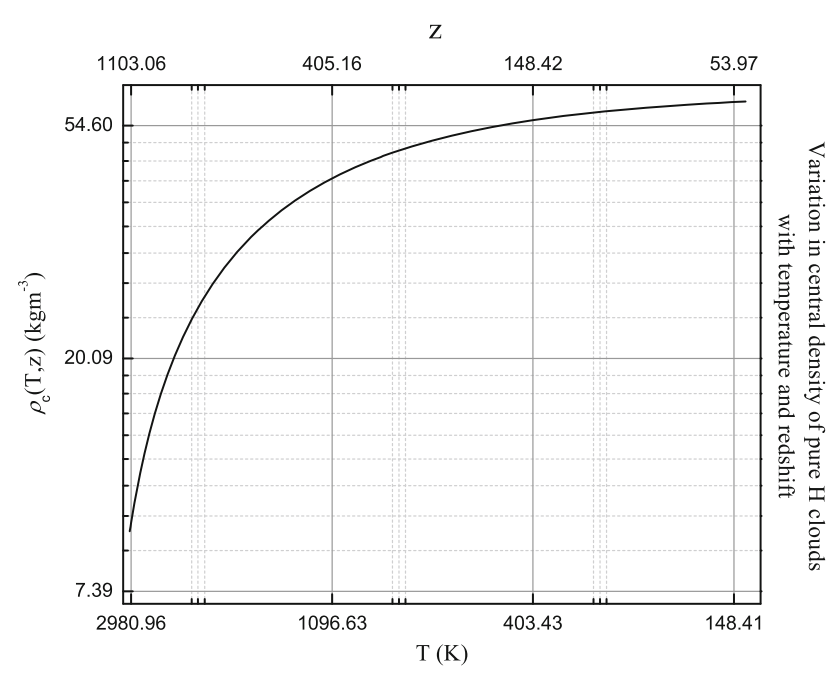

Fig. 1 Variation in the central density $\rho_{c}$ of the virial clouds with respect to both the red shift (indicated by the upper horizontal axis) and the $\mathrm{CMB}$ temperature (bottom axis) for pure $\mathrm{H}$ clouds

and

$r \frac{d \rho(r)}{d r}-r^{2}\left(\frac{4 \pi G m}{k_{B} T}\right) \rho^{2}(r)-\rho(r) \ln \left(\frac{\rho(r)}{B}\right)=0$,

where, $B=\left(8 m^{5 / 2} / 3^{3 / 2}\right)\left(G \rho_{c} / k_{B} T\right)^{3 / 2}, m$ is the mass of hydrogen atom, $1.67 \times 10^{=27} \mathrm{~kg}, G$ is Newton's gravitational constant, $\rho_{c}$ is the central density of the cloud, and $M(r)=$ $\int_{0}^{r} 4 \pi \rho(q) q^{2} d q$ is the mass interior to $r$.

The above expressions gives us the central density of the cloud. Now we need the Jeans mass and radius. From the virial theorem, $2 K+\Phi=0$, where, $K$ is the kinetic energy of the molecules and $\Phi$ is the gravitational potential, the Jeans mass squared is [27]

$M_{J}^{2} \simeq\left(\frac{81}{32 \pi \rho_{c}}\right)\left(\frac{k_{B} T}{G m}\right)^{3}$,

and the corresponding Jeans length (radius) squared is

$R_{J}^{2}=\frac{27 k_{B} T}{20 \pi \rho_{c} G m}$.

We use Eq. (2) to estimate the central density of the clouds. We solve the equation numerically with a guess value of $\rho_{c}$, at a fixed temperature, and see where the density becomes exactly zero at the boundary. We then compare the Jeans radius with that central density with the value available to us. We adjust the central density so that the density becomes zero exactly at the Jeans radius. In this way we get a selfconsistent solution of the differential equation subject to the given boundary conditions. Next, we decrease the temperature and repeat the process. The evolution of the central density of these clouds as a function of both the time and the CMB temperature is shown in Fig. 1 the density of these clouds tend to increase with the decrease in temperature.

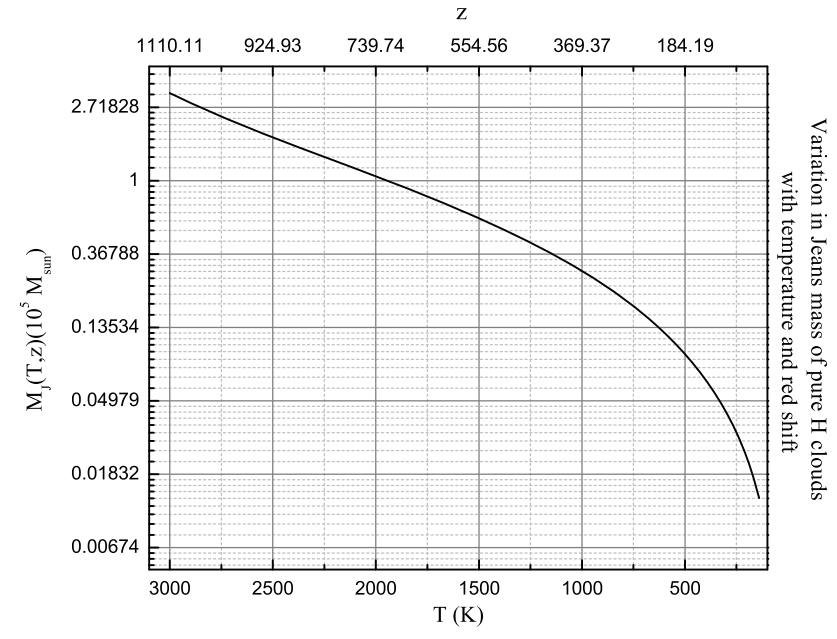

Fig. 2 Variation in the Jeans mass of pure $\mathrm{H}$ virial clouds

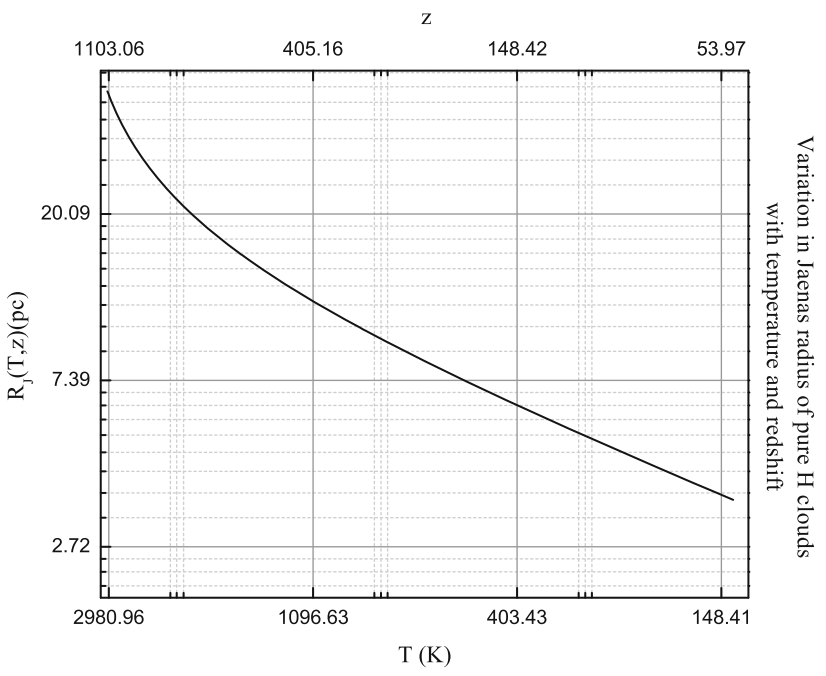

Fig. 3 Variation in the Jeans radius of pure $\mathrm{H}$ virial clouds

We also give the evolution of the Jeans mass (see Fig. 2) and radius (see Fig. 3 ) of pure $\mathrm{H}$ virial clouds.

\subsection{Two-fluid virial cloud}

For generality we consider a virial cloud composed of an arbitrary mixture of $\mathrm{H}$ and $\mathrm{He}$, with mass fractions $\alpha$ and $\beta$. Then, we use the primordial cosmological fractions of $\mathrm{H}$ and $\mathrm{He}$ for the final computation. The total mass of the cloud is, obviously, $M_{c l}(r)=\alpha M_{H}(r)+\beta M_{H e}(r)$, with the condition $\alpha+\beta=1$. The density distribution for two fluids is given by [15]

$\rho_{c l}(r)=\sqrt{\frac{64}{27}} \frac{\left(G \rho_{c_{H}} \rho_{c_{H e}}\right)^{3 / 2}}{\left(k T_{C M B}\right)^{9 / 2}}\left(m_{H} m_{H e}\right)^{5 / 2}$ 


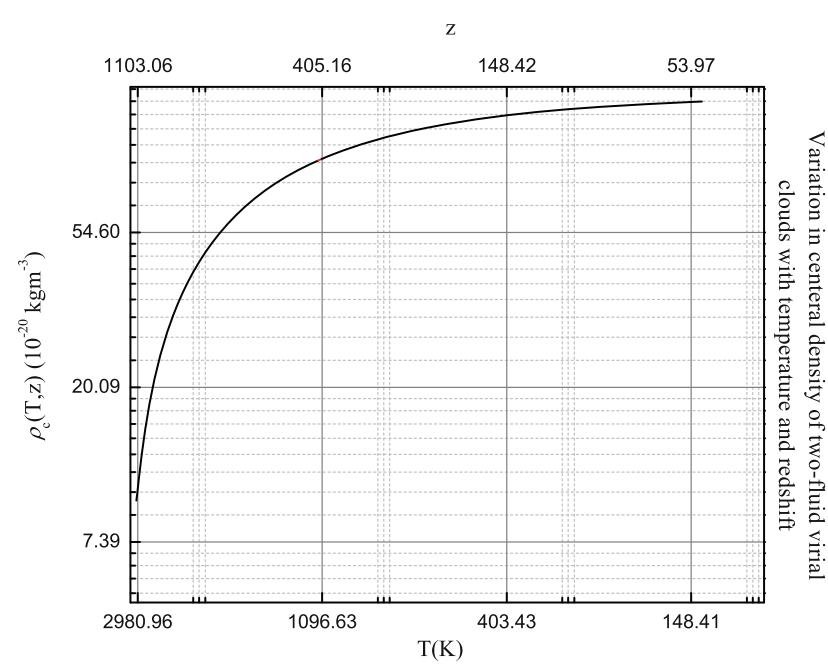

Fig. 4 Variation in the central density of the virial clouds made up by a mixture of $\mathrm{H}$ and $\mathrm{He}$ with cosmological primordial relative abundances

$$
\times \exp \left[-\frac{1}{2}\left(\frac{\alpha G M_{H}(r) m_{H}}{r k_{B} T}+\frac{\beta G M_{H e}(r) m_{H e}}{r k_{B} T}\right)\right] .
$$

and the corresponding Lane-Emden equation is

$$
\begin{aligned}
& r \frac{d \rho_{c l}(r)}{d r}-r^{2}\left(\frac{2 \pi G}{k_{B} T}\right)\left[\rho _ { c l } ( r ) \left(\alpha \rho_{c_{H}} m_{H}\right.\right. \\
& \left.\left.\quad+\beta \rho_{c_{H e}} m_{H e}\right)\right]-\rho_{c l}(r) \ln \left(\frac{\rho_{c l}(r)}{\tau}\right)=0,
\end{aligned}
$$

where, $\tau=(8 / 3 \sqrt{3})\left[\left(G \rho_{c_{H}} \rho_{c_{H e}}\right)^{3 / 2} /\left(k_{B} T\right)^{9 / 2}\right]\left[m_{H} m_{H e}\right]^{5 / 2}$, $\rho_{c_{H}}$ is the central density of H cloud, $\rho_{c_{H e}}$ is the central density of He cloud, $m_{H}$, the mass of single atom of hydrogen, and $m_{H e}$, the mass of single atom of helium.

Using the same approach we used to solve the LaneEmden equation for a single fluid, we estimated the central density, Jeans mass and radius of the two-fluid virial clouds with primordial fraction of $\mathrm{H}$, and He, i.e. $\alpha=0.75$, and $\beta=0.25$.

The evolution of the central density of the two-fluid $\mathrm{H}$ He virial cloud, both with respect to the time and the CMB temperature, is shown in Fig. 4.

Similarly, the evolution of the Jeans mass is shown in Fig. 5 while the evolution of Jeans radius is shown in Fig. 6. As one can see, the physical parameters of the two-fluid clouds do not change substantially with respect to the pure $\mathrm{H}$ clouds.

\section{Results and discussion}

In Sect. 2 it has been shown that the evolution of the virial clouds is precisely determinable from the SLS up to the for-

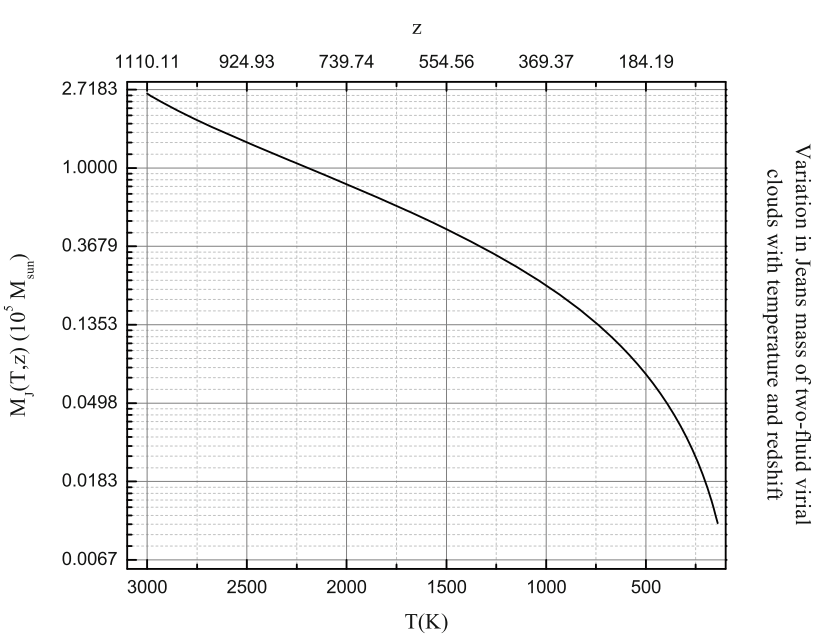

Fig. 5 Variation in the Jeans mass of the virial clouds constituted by cosmological primordial fraction of $\mathrm{H}$ and $\mathrm{He}$

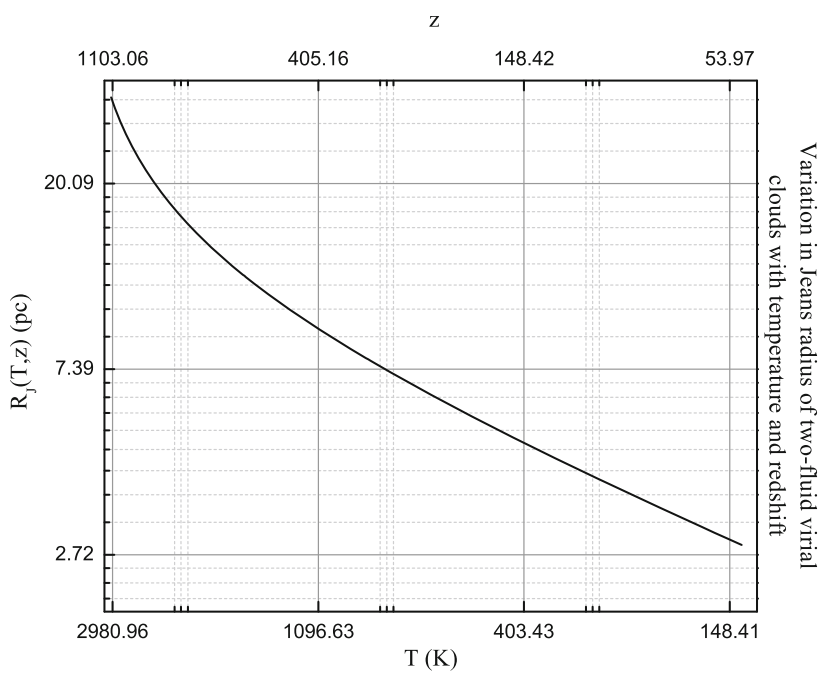

Fig. 6 Variation in the Jeans radius of the virial clouds having primordial fraction of $\mathrm{H}$ and $\mathrm{He}$

mation of population-III stars. In Figs. 1, 2 and 3 we saw that pure $\mathrm{H}$ clouds would have been more massive, larger and less dense in the beginning, and would have lost their mass and size as the temperature decreased, in such a way that their density increased. In the case of clouds constituted by two fluids with a cosmological primordial mixture of $\mathrm{H}$ and $\mathrm{He}$, the evolution of the cloud physical parameters does not change substantially with respect to the single fluid model: the cloud central density tends to increase while the Jeans mass and the Jeans radius decrease with the CMB temperature (see Figs. 4, 5 and 6). However, with a more detailed analysis of Figs. 4, 5 and 6 with respect to Figs. 1, 2 and 3 , one can note that the addition of $\mathrm{He}$ in the clouds makes them slightly denser, smaller and less massive with respect to the pure $\mathrm{H}$ cloud. This is because of the stronger gravitational force for the heavier molecules, but the average effect 
in the parameters is nearly the same as the single fluid $\mathrm{H}$ virial clouds.

Since we are dealing with an era from $\mathrm{z}=1100$ to $\mathrm{z}=50$, one might expect that there would be gravitational clumping in the dark matter gravitational potential well, where there will be gravitational binding energy and hence cooling, so that molecular hydrogen would be more likely to form. The $\mathrm{H}_{2}$ component would be negligible in any case, but more so as there would be no nucleating particles for it in the absence of dust grains to speed up the formation of the molecules. If any $\mathrm{H}_{2}$ molecule formed during this era it was dissociated by the radiation until the density low enough for them to be stable [28]. Hence we do not need to consider the effect of cooling from molecular hydrogen till population-III stars exploded.

It might be thought that quantum calculations for the scattering cross-section of photon molecule interaction need to be incorporated. We checked the electronic transitions of hydrogen atoms due to CMB photons at $3000 \mathrm{~K}$. Most of the wavelengths in the Planck spectrum at $3000 \mathrm{~K}$ do not have enough energy to excite the electron of an $\mathrm{H}$ atom from its ground state, but the higher energy tail does have sufficient energy to do so. The percentage of such photons is low but it is not zero. The energy of the second orbit of hydrogen is $3.41 \mathrm{eV}$, so the CMB photons with the same energy or higher can take part in the transition. The total number density of photons at $3000 \mathrm{~K}$ is $5.47 \times 10^{17} \mathrm{~m}^{-3}$ and the number density of photons between 0 to $365 \mathrm{~nm}$ at the same temperature is $8.96 \times 10^{13} \mathrm{~m}^{-3}$, hence the percentage of high energy photon is 0.016 , which is quite low. Hence, there will be a negligible effect of high energy photon which will cause atomic transitions in hydrogen atoms, so we do not need to consider the quantum effects at this stage, but these effects might have a sufficient effect when heavier atoms or molecules contaminate the clouds. These things will be discussed in the second step.

One may wonder if the model presented is not oversimplistic as it ignores any possible rotation of the clouds and any turbulence that may arise or be present in the medium during the formation stage of the virial clouds [29,30]. Our whole purpose in limiting the discussion in this first paper to the time before population III stars form was to keep the Physics simple without having to neglect some other effects. Unless there is some primordial angular momentum present at the surface of last scattering, there would have to be a mechanism for angular momentum to be generated. The natural mechanism for it to arise in the early stages of galaxy and star formation would be that significant amounts of ejecta from stars, collide with a non-zero impact parameter (as it is most natural to happen), thereby producing local angular momentum. However, that could only occur after the very first stars, i.e. population III stars, explode. This would not only produce the angular momentum that was to be gener- ated locally, it would also produce turbulence and lead to contamination of the clouds by matter that is not primordial. This will have to be investigated in the next paper that will trace the evolution from the formation of population III stars on. For this purpose one would need to estimate how much angular momentum would get generated and whether, and to what extent, it would lead to a rotation of the clouds. Again, one would need to investigate how much turbulence would be generated in the earlier stages of the formation of the clouds, and whether, and to what extent, it would enter into the virial clouds At present it is anticipated that both effects will be minor, and will be amenable to inclusion as small corrections on the basic model. Some effects, such as the inclusion of higher modes in the virial clouds, which would need to use quantum calculations, will occur. Similarly, contamination of the clouds by other matter will occur. Since the mechanism for the new effects is expected to be driven by population III stars, it might be possible to test the model by looking for a correlation between the small-scale anisotropies in the $\mathrm{CMB}$ and the distribution of population III stars.

Till the explosion of Population III stars no other fluids or substantial contaminants should be present in the virial clouds. However, for the subsequent steps of the cloud evolution we will need to include more fluids (heavier atoms) and dust grains produced by population III stars and ejected into the ISM through their explosions. These dust grains should have an important role in the cloud evolution since they would act as catalysts for the formation of molecular hydrogen [31], possibly leading to rapid changes in the cloud physical parameters, as higher modes would be excited. In any case, the procedure we have set up in the previous section is not limited to the two fluids case and would be able to accommodate more fluids when the need arises. Of course, this first step was the simplest one since the composition of the Universe from the SLS to the explosion of Population III stars is precisely known. The Physics involved is also simple as the temperature is comparatively high and quantum effects can be neglected. In the next stage ambiguity arises as the cloud chemical composition becomes more complicated and less well known. However, the Physics is relatively simple. At this stage we will have to incorporate the quantum calculations that were found to be negligible in the present paper.

Acknowledgements FDP and NT acknowledge the TAsP and Euclid INFN projects. We are most grateful to the anonymous referee for the constructive and useful suggestions, which are particularly relevant for the analysis of the following stages of the virial clouds evolution.

Data Availability Statement This manuscript has no associated data or the data will not be deposited. [Authors' comment: This is a theoretical study and there is no data to be associated with it, hence we do not need to list any experimentaal data.]

Open Access This article is licensed under a Creative Commons Attribution 4.0 International License, which permits use, sharing, adaptation, 
distribution and reproduction in any medium or format, as long as you give appropriate credit to the original author(s) and the source, provide a link to the Creative Commons licence, and indicate if changes were made. The images or other third party material in this article are included in the article's Creative Commons licence, unless indicated otherwise in a credit line to the material. If material is not included in the article's Creative Commons licence and your intended use is not permitted by statutory regulation or exceeds the permitted use, you will need to obtain permission directly from the copyright holder. To view a copy of this licence, visit http://creativecomm ons.org/licenses/by/4.0/.

Funded by SCOAP 3 .

\section{References}

1. F. De Paolis et al., Phys. Rev. Lett. 74, 14 (1995)

2. F. De Paolis et al., Astron. Astrophys. 299, 647 (1995)

3. F. De Paolis et al., Astron. Astrophys. 534, L8 (2011)

4. F. De Paolis et al., Astron. Astrophys. 565, L3 (2014)

5. F. De Paolis et al., J. Phys: Conf. Ser. 354, 1 (2012)

6. F. De Paolis et al., Astron. Astrophys. 580, L8 (2015)

7. V. Gurzadyan et al., Astron. Astrophys. 582, A77 (2015)

8. F. De Paolis et al., Astron. Astrophys. 593, A57 (2016)

9. V. Gurzadyan et al., Astron. Astrophys. 609, A131 (2018)

10. F. De Paolis et al., Astron. Astrophys. 629, A87 (2019)

11. N. Tahir et al., Int. J. Mod. Phys. D 28, 7 (2019)

12. N. Tahir et al., Arab. J. Math. 8, 193 (2019)
13. A. Qadir et al. in Proceed. of the 15th Marcel Grossman Meeting, 2019, ed. by, R. Ruffini, R.T. Jantzen (World Scientific, 2019) (to appear)

14. T. Padmanabhan, Phys. Rep. 188, 285 (1990)

15. A. Qadir et al., Phys. Rev. D 100, 043028 (2019)

16. R. Barkana et al., Phys. Rep. 349, 125 (2001)

17. J. Miralda-Escudé, Science 300, 5627 (2003)

18. N. Yoshida et al., Astrophys. J. 605, 579 (2004)

19. M. Jeon et al., Mon. Not. R. Astron. Soc. 444, 3288 (2014)

20. P.J.E. Peebles, Astrophys. J. 146, 542 (1966)

21. R.V. Wagoner et al., Astrophys. J. 148, 3 (1967)

22. A. Friedman, Z. Angew. Phys. 10, 377 (1922)

23. S. Lepp et al., Astrophys. J. 280, 465 (1984)

24. P.J.E. Peebles et al., Astrophys. J. 154, 891 (1968)

25. W.C. Saslaw et al., Nature 216, 976 (1967)

26. P.M. Morse et al., Phys. Today 17, 60 (1964)

27. S. Chandrasekhar, An introduction to the study of stellar structure, vol. 2 (Courier Corporation, 1957)

28. S.C. William et al., Nature 216, 5119 (1967)

29. A. Dekel et al., Astrophys. J. 703, 785 (2009)

30. E. Rosolowsky et al., Astophys. J. 599, 278 (2003)

31. B.M. Tinsley, Fund. Cosm. Phys. 5, 287 (1980) 